\title{
AN EXISTENCE THEOREM FOR ORDINARY DIFFERENTIAL EQUATIONS IN BANACH SPACES ${ }^{1}$
}

\author{
BY SHUI-NEE CHOW AND J. D. SCHUUR
}

Communicated by Fred Brauer, May 24, 1971

Abstract. We consider nonlinear ordinary differential equations in Banach spaces. A local existence theorem for the Cauchy problem is given when the equation is continuous in the weak topology. The theorem can be extended to set-valued differential equations in Banach spaces.

Let $B$ be a Banach space and let $F:(0,1) \times B \rightarrow B$. If $B$ is finite dimensional and $F$ is continuous in a neighborhood of $\left(t_{0}, x_{0}\right) \in(0,1)$ $\times B$, then by the Peano existence theorem there exists a function $\phi(t)$ defined on a subinterval of $(0,1)$ such that

$$
\phi^{\prime}(t)=F(t, \phi(t)) \text { and } \phi\left(t_{0}\right)=x_{0} .
$$

Dieudonné [1] and Yorke [2] have shown, by means of examples, that continuity alone, of the function $F$, is not sufficient to prove a local existence theorem in the case where $B$ is infinite dimensional. Other authors, for example [3] and [4], have extended the Peano theorem to infinite-dimensional spaces but with additional assumptions. We have found that by replacing strong continuity with weak continuity and assuming the range of $F$ to be bounded we may obtain an existence theorem.

Let $B$ be a separable reflexive Banach space with norm $\|\cdot\|$ and let $B^{*}$ be its dual space. Let $B_{w}$ denote the space $B$ with the weak topology and let $\left\{f_{i}\right\}$ be a countable dense subset in $B^{*}$. By $\Delta$ we mean a subinterval of $T=(0,1)$.

Definition 1. A function $F: T \times B \rightarrow B$ is said to satisfy condition (I) if, at each $\left(t_{0}, x_{0}\right) \in T \times B$,

$$
F\left(t_{0}, x_{0}\right)=\bigcap_{N=1}^{\infty} \operatorname{cl} \operatorname{co} F_{N}\left(t_{0}, x_{0}\right)
$$

where

AMS 1969 subject classifications. Primary 3495, 3404; Secondary 2630.

Key words and phrases. Ordinary differential equations, Banach spaces, weak topology, existence of solution, set-valued differential equation, Cesari upper semicontinuity.

1 This work was partially supported by the National Science Foundation under contract NSF GU-2648 and the Office of Naval Research under contract N000-1468-A-0109-005. 


$$
\begin{aligned}
F_{N}\left(t_{0}, x_{0}\right)=\bigcup\left\{F(t, x):\left|t-t_{0}\right|<\frac{1}{N}\right. \\
\\
\left.\qquad f_{i}\left(x-x_{0}\right) \mid<\frac{1}{N}, i=1, \cdots, N\right\}
\end{aligned}
$$

and cl co $F_{N}\left(t_{0}, x_{0}\right)$ denotes the closed convex hull of $F_{N}\left(t_{0}, x_{0}\right)$.

If $B$ is finite dimensional, condition (I) is equivalent to saying that $F$ is continuous. If $F: T \times B_{w} \rightarrow B_{w}$ is continuous, then $F$ satisfies condition (I). If $F$ takes on set values in $B$ and $B$ is finite dimensional, then condition (I) is equivalent to saying that $F$ is upper semicontinuous in the sense of Cesari [5], [6]. Thus, condition (I) is a reasonable generalization of continuity and it will also apply to set-valued differential equations.

We consider the differential equation

$$
\dot{x}=F(t, x), \text { where } F: T \times B \rightarrow B .
$$

Definition 2. A solution of (E) on $\Delta$ is a function $\phi(t)$ defined on $\Delta$ such that $\phi(t)$ is weakly continuous (i.e., for every $f \in B^{*}, f(\phi(t))$ is a continuous real-valued function), and for almost every $t \in \Delta$,

$$
D \phi(t)=F(t, \phi(t))
$$

where $D \phi(t)$ is the weak limit of $(\phi(t+h)-\phi(t)) / h$ as $h \rightarrow 0$.

THEOREM 1. Let $F: T \times B \rightarrow B$ satisfy condition (I) and let $\phi: \Delta \rightarrow B$ be weakly continuous. Then $\phi(t)$ is a solution of $(\mathrm{E})$ on $\Delta$ if and only if, for every $N \geqq 1, t \in \Delta$, there exists $\eta>0$ such that

$$
0<h<\eta \Rightarrow \frac{\phi(t+h)-\phi(t)}{h} \in \operatorname{cl} \operatorname{co} F_{N}(t, \phi(t)) .
$$

Theorem 2. Let $F: T \times B \rightarrow B$ and let $\left(t_{0}, x_{0}\right) \in T \times B$. Assume that in a neighborhood of $\left(t_{0}, x_{0}\right), F$ satisfies condition (I) and is bounded in norm. Then there exists a solution $\phi(t)$ of $(\mathrm{E})$ on some interval $\Delta$ such that $\phi\left(t_{0}\right)=x_{0}$. Further, $\phi(t)$ is absolutely continuous and $\phi^{\prime}(t)=$ $F(t), \phi(t))$ a.e. on $D$ (where $\phi^{\prime}(t)$ is the strong limit of $(\phi(t+h)-\phi(t)) / h$ as $h \rightarrow 0$ ).

The method of polygonal approximations is used in this proof. The approximations converge weakly to a weakly continuous limit function. By Theorem 1, we are able to show that this function is a solution of (E) and that it is Lipschitz. Then by a result of Pettis [7] it follows that $\phi$ has a strong derivative a.e. 
Corollary. Let $F: T \times B_{w} \rightarrow B_{w}$ and let $\left(t_{0}, x_{0}\right) \in T \times B_{w}$. Assume that in a neighborhood of $\left(t_{0}, x_{0}\right), F$ is continuous and bounded in norm. Then there exists a solution $\phi(t)$ of $(\mathrm{E})$ defined on some interval $\Delta$ such that $\phi\left(t_{0}\right)=x_{0}, \phi(t)$ is absolutely continuous on $\Delta$, and $\phi^{\prime}(t)=F(t, \phi(t))$ a.e. on $\Delta$.

We have also proved Theorem 2 in the case where $F$ takes values in the closed convex subsets of $B$ (i.e. for set-valued differential equations) and we have proved theorems on continuous dependence on initial conditions and closure of families of solutions.

\section{REFERENCES}

1. J. Dieudonné, Deux exemples singuliers d'équations différentielles, Acta Sci. Math. Szeged (Leopoldo Fejér Frederico Riesz LXX annus natis dedicatus, pars B) 12 (1950), 38-40. MR 11, 729.

2. J. A. Yorke, $A$ continuous differential equation in Hilbert space without existence, Funkcial Ekvac. 13 (1970), 19-21.

3. M. A. KrasnoselskiY and S. G. Kreln, Nonlocal existence theorems and uniqueness theorems for systems of ordinary differential equations, Dokl. Akad. Nauk SSSR 102 (1955), 13-16. (Russian) MR 17, 151.

4. C. Corduneanu, Equazioni differenziali negli spazi di Banach, Teoremi di esistenze e di prolungabilita, Atti Accad. Naz. Lincei Rend. Cl. Sci. Fiz. Mat. Nat. (8) 23 (1957), 226-230. MR 20 \#3312.

5. L. Cesari, Existence theorems for optimal solutions in Pontryagin and Lagrange problems, SIAM. J. Control 3 (1966), 475-498.

6. A. Lasota and C. Olech, On Cesari's semicontinuity condition for set valued mappings, Bull. Acad. Polon. Sci. Sér. Sci. Math. Astronom. Phys. 16 (1968), 711-716. MR 39 \#6138.

7. B. J. Pettis, A note on regular Banach spaces, Bull. Amer. Math. Soc. 44 (1938), $420-428$.

Michigan State University, East Lansing, Michigan 48823 